Swarthmore College

Works

$5-1-2004$

\title{
Tracer Spectroscopy Diagnostics Of Doped Ablators In Inertial Confinement Fusion Experiments On Omega
}

David H. Cohen

Swarthmore College, dcohen1@swarthmore.edu
J. J. MacFarlane
P. Jaanimagi
O. L. Landen
D. A. Haynes

See next page for additional authors

Follow this and additional works at: https://works.swarthmore.edu/fac-physics

Part of the Astrophysics and Astronomy Commons

Let us know how access to these works benefits you

\section{Recommended Citation}

David H. Cohen; J. J. MacFarlane; P. Jaanimagi; O. L. Landen; D. A. Haynes; David Scott Conners , '03; Katherine Lenore Penrose , '04; and Nathan Cooper Shupe, '05. (2004). "Tracer Spectroscopy Diagnostics Of Doped Ablators In Inertial Confinement Fusion Experiments On Omega". Physics Of Plasmas. Volume 11, Issue 5. 2702-2708. DOI: 10.1063/1.1647135

https://works.swarthmore.edu/fac-physics/53

This work is brought to you for free by Swarthmore College Libraries' Works. It has been accepted for inclusion in Physics \& Astronomy Faculty Works by an authorized administrator of Works. For more information, please contact myworks@swarthmore.edu. 


\section{Authors}

David H. Cohen; J. J. MacFarlane; P. Jaanimagi; O. L. Landen; D. A. Haynes; David Scott Conners , '03;

Katherine Lenore Penrose , '04; and Nathan Cooper Shupe , '05 


\section{AIP | Physics of Plasmas}

Tracer spectroscopy diagnostics of doped ablators in inertial confinement fusion experiments on OMEGA

David H. Cohen, Joseph J. MacFarlane, Paul Jaanimagi, Otto L. Landen, Donald A. Haynes, David S. Conners, Katherine L. Penrose, and Nathan C. Shupe

Citation: Physics of Plasmas (1994-present) 11, 2702 (2004); doi: 10.1063/1.1647135

View online: http://dx.doi.org/10.1063/1.1647135

View Table of Contents: http://scitation.aip.org/content/aip/journal/pop/11/5?ver=pdfcov

Published by the AIP Publishing

\section{AlP}

\section{Create a profile. 圆 Sign up today!}




\title{
Tracer spectroscopy diagnostics of doped ablators in inertial confinement fusion experiments on OMEGA ${ }^{\text {a) }}$
}

\author{
David H. Cohen ${ }^{\text {b) }}$ \\ Department of Physics and Astronomy, Swarthmore College, Swarthmore, Pennsylvania 19081 \\ Joseph J. MacFarlane \\ Prism Computational Sciences, 455 Science Drive, Suite 140, Madison, Wisconsin 53711 \\ Paul Jaanimagi \\ Laboratory for Laser Energetics, University of Rochester, Rochester, New York 14627 \\ Otto L. Landen \\ Lawrence Livermore National Laboratory, Livermore, California 94550 \\ Donald A. Haynes \\ Los Alamos National Laboratory, Los Alamos, New Mexico 87545
}

David S. Conners, Katherine L. Penrose, and Nathan C. Shupe

Department of Physics and Astronomy, Swarthmore College, Swarthmore, Pennsylvania 19081

(Received 27 October 2003; accepted 11 December 2003; published online 23 April 2004)

\begin{abstract}
A technique has been developed for studying the time-dependent, local physical conditions in ablator samples in an inertial confinement fusion (ICF) hohlraum environment. This technique involves backlit point-projection absorption spectroscopy of thin tracer layers buried in the interior of solid samples mounted on laser-driven hohlraums. It is shown how detailed view-factor, atomic, hydrodynamics, and radiation-transport modeling can be used to infer time-dependent physical conditions in the interiors of these samples from the observed absorption spectra. This modeling is applied to the results of an experimental campaign on the OMEGA laser [T. R. Boehly et al., Opt. Commun. 133, 495 (1997)] designed to compare radiation-wave velocities in doped and undoped ICF ablator materials. (C) 2004 American Institute of Physics. [DOI: 10.1063/1.1647135]
\end{abstract}

\section{INTRODUCTION}

The efficient compression of an indirect-drive inertial confinement fusion (ICF) fuel capsule depends sensitively on the coupling of the hohlraum $\mathrm{x}$-ray drive to the capsule ablator. In order to control the deposition of energy in the ablator, and thus the shock compression of the fuel, mid-Z ablator dopants such as germanium, bromine, or copper are often added to the low-Z ablator material. ${ }^{1,2}$ The increased opacity provided by ablator dopants (see Fig. 1) serves to change the radiation-matter coupling as well as to shield the fuel from preheat. It is desirable for experimentalists to be able to monitor the effects of ablator dopants on the physical conditions within a capsule ablator exposed to a hohlraum x-ray drive. In the past, the effects of ablator dopants have been studied via imaging of imploded capsules ${ }^{1}$ and via shock breakout or burnthrough and foil motion measurements. ${ }^{3,4}$ In these cases, the integrated effects of the radiation hydrodynamics over the course of a capsule implosion, burnthrough, etc., are diagnosed. In this paper we report on experiments and associated modeling undertaken to study the effects of dopants at a specific location in the interior of an ablator sample. This technique, which involves

\footnotetext{
a) Paper FI2 5, Bull. Am. Phys. Soc. 48, 86 (2003).

${ }^{b)}$ Invited speaker. Electronic mail: dcohen1@ swarthmore.edu
}

point-projection spectroscopy of a thin tracer layer, could be generally applied to the study of material samples exposed to strong radiation fields.

It has previously been shown that $\mathrm{K}_{\alpha}$ absorption spectroscopy can be used to study energy transport ${ }^{5}$ and material properties such as opacities. ${ }^{6,7}$ Tracer emission in hohlraums ${ }^{8}$ and from witness plates mounted on hohlraums ${ }^{9}$ has also been employed as a diagnostic of physical conditions in hohlraum plasmas. Additionally, Chenais-Popovics et al. ${ }^{10}$ have shown that chlorine $\mathrm{K}_{\alpha}$ absorption spectroscopy, backlit by bismuth M-shell emission, is a useful diagnostic of laserproduced plasmas.

We have combined these ideas in developing a program to study the time-dependent conditions within ablator samples mounted on halfraums at the OMEGA laser facility. ${ }^{11}$ We use thin (submicron) chlorine-bearing tracer layers buried at known depths within these ablator samples to diagnose the ionization state (and thus localized plasma conditions) as a function of time at depths of several microns in these samples. Ultimately, these data reflect the Marshak wave velocity in the ablators. Modeling of the experiments using view factor, radiation hydrodynamics, and spectral synthesis codes is an important component of the program, allowing us to infer physical conditions from spectral measurements and giving us greater confidence in making comparisons between doped and undoped ablator samples.

In the experiments we mounted planar ablator samples 


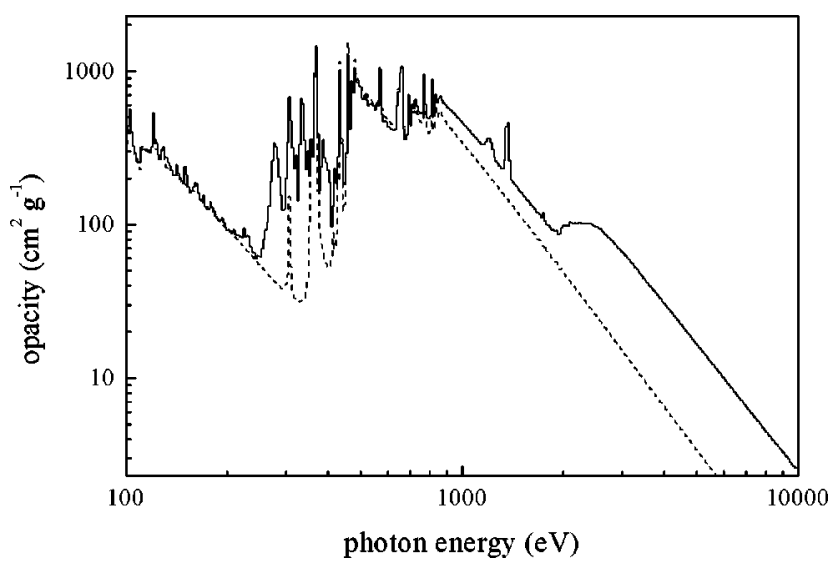

FIG. 1. Comparison of the opacity of undoped (dashed) and doped (solid) plastic ablators at $\mathrm{k} T=100 \mathrm{eV}$ and $n_{\text {ion }}=10^{21} \mathrm{~cm}^{-3}$. The dopant is germanium at $1.8 \%$ atomic.

on the back ends of halfraums, which we shot on OMEGA in a standard configuration. We used planar samples for the sake of simplified hydrodynamics, and model the radiation drive onto the samples, which, in any case, is very similar to what a spherical capsule in a hohlraum sees. At this point we do not have plans to attempt this measurement in an actual spherical pellet, although this would be possible as long as the backlighter $\mathrm{x}$ rays were not too attenuated by the material in the pellet. The drive modeling can, in principle, be constrained by absolutely calibrated DANTE ${ }^{12}$ measurements of the halfraum wall reemission. However, we have found that in general, the radiation field inside the halfraum must be modeled, as it varies spectrally with location as well as time.

The planar ablator samples are specially fabricated to have a thin (roughly $0.4 \mu \mathrm{m}$ ) $\mathrm{NaCl}$ layer about $5 \mu \mathrm{m}$ below the surface. This chlorine-bearing tracer gives rise to a $\mathrm{K}_{\alpha}$ absorption signal just below $2800 \mathrm{eV}$ seen in absorption against a laser-produced bismuth plasma generated by diverting several beams onto a Bi foil hung off the halfraum in front of the laser entrance hole (LEH). See Fig. 2 for an image and model of a target. ${ }^{18}$ We used the LLE X-ray spectrometer (LXS) coupled to a streak camera to make timedependent measurements of the tracer spectral signal.

Our modeling, which is described in detail in Sec. III, shows that the $\mathrm{Cl} \mathrm{K}_{\alpha}$ features turn on when the Marshak wave reaches the tracer layer and heats the tracer to roughly $100 \mathrm{eV}$. The shock wave driven in front of the Marshak wave does not heat the tracer enough to ionize up to the L-shell, which is required for the production of $\mathrm{K}_{\alpha}$ absorption. If the effect of ablator dopants is to reduce the Marshak wave velocity, as is expected, then one should see the tracer signal turn on earlier in an undoped ablator sample compared to a doped sample (in cases where the tracer depth is the same in the two samples). This is demonstrated by the model spectra time series shown in Fig. 3. A second-order effect that one can also observe is the time-dependent ionization balance in the tracer, which reflects changing temperature and density conditions at the location of the tracer as the Marshak wave passes through.
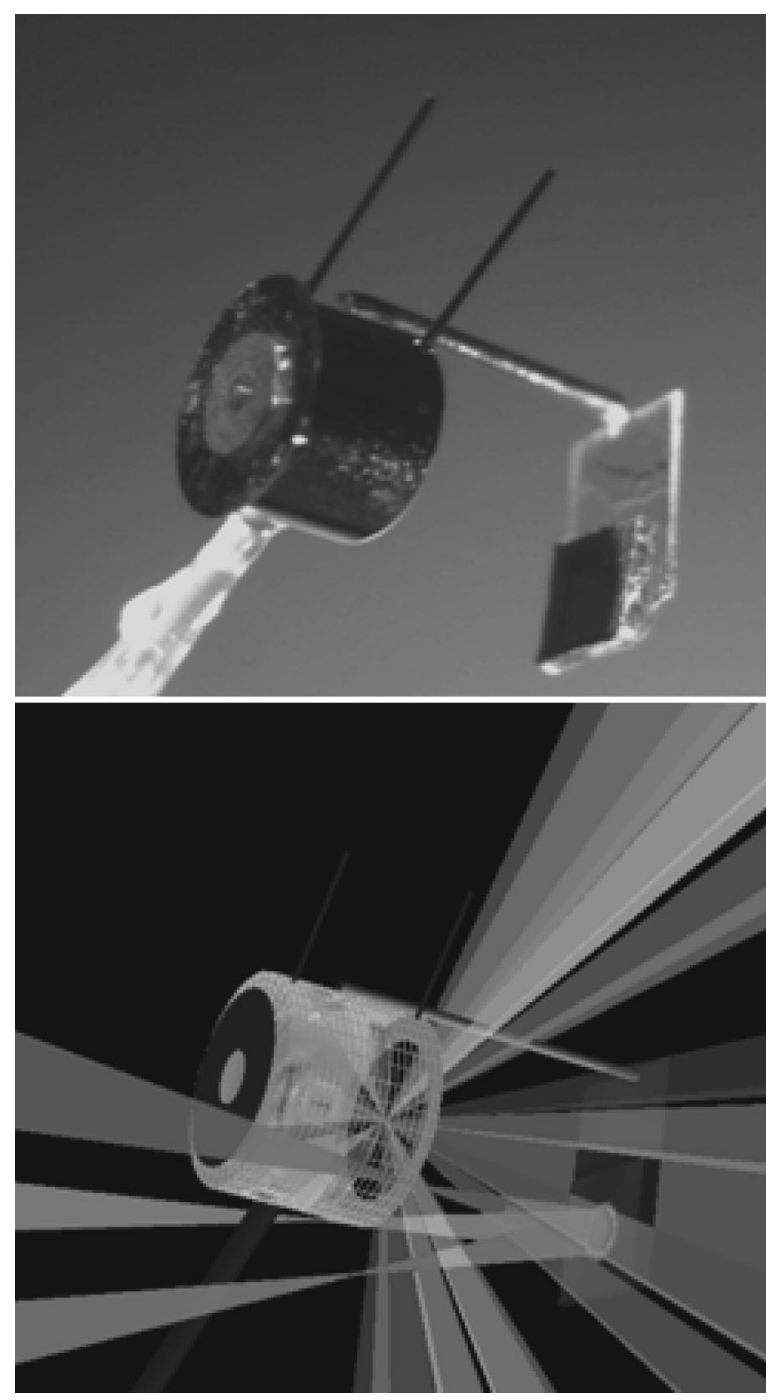

FIG. 2. A photograph of one of our halfraum targets (top), taken in the metrology lab at the Laboratory for Laser Energetics (LLE). The laser entrance hole (LEH) is (obscured) on the right end of the cylinder and the ablator sample is mounted on the opposite end, apertured by a washer. The backlighter foil is visible in front of the LEH. It is mounted on lead-doped plastic to block hot backlighter plasma blow-off from the DANTE line of sight. The stalk which holds the target is visible on the lower left of the frame and the two wires on the top of the halfraum barrel facilitate the alignment process in the target chamber. The lower panel shows a model of the target, made with the VISRAD view-factor code (Ref. 13) (described in Sec. III). The 15 target laser beams and 3 backlighter beams are shown in this simulation. The target is shaded according to the calculated electron temperature. Note the laser hot spots. The near wall of the halfraum is depicted as a mesh frame to allow us to see the interior, which makes the LEH visible in this image.

\section{EXPERIMENTS AND DATA}

We performed a series of shots on the OMEGA laser as part of the National Laser Users Facility (NLUF) program, comparing doped and undoped ablator samples on scale-1 halfraums mounted on the P6-P7 axis. We used 15 cone 2 and cone 3 beams, pointed at the center of the LEH (giving laser spot positions on the wall ranging from 480 to $890 \mu \mathrm{m}$ from the LEH plane), for a total of approximately $6.7 \mathrm{~kJ}$ delivered in a $1 \mathrm{~ns}$ square pulse. Unfolded DANTE measurements indicated peak wall reemission temperatures of 182 $\mathrm{eV}$, with the two shots we compare here both peaking at this 

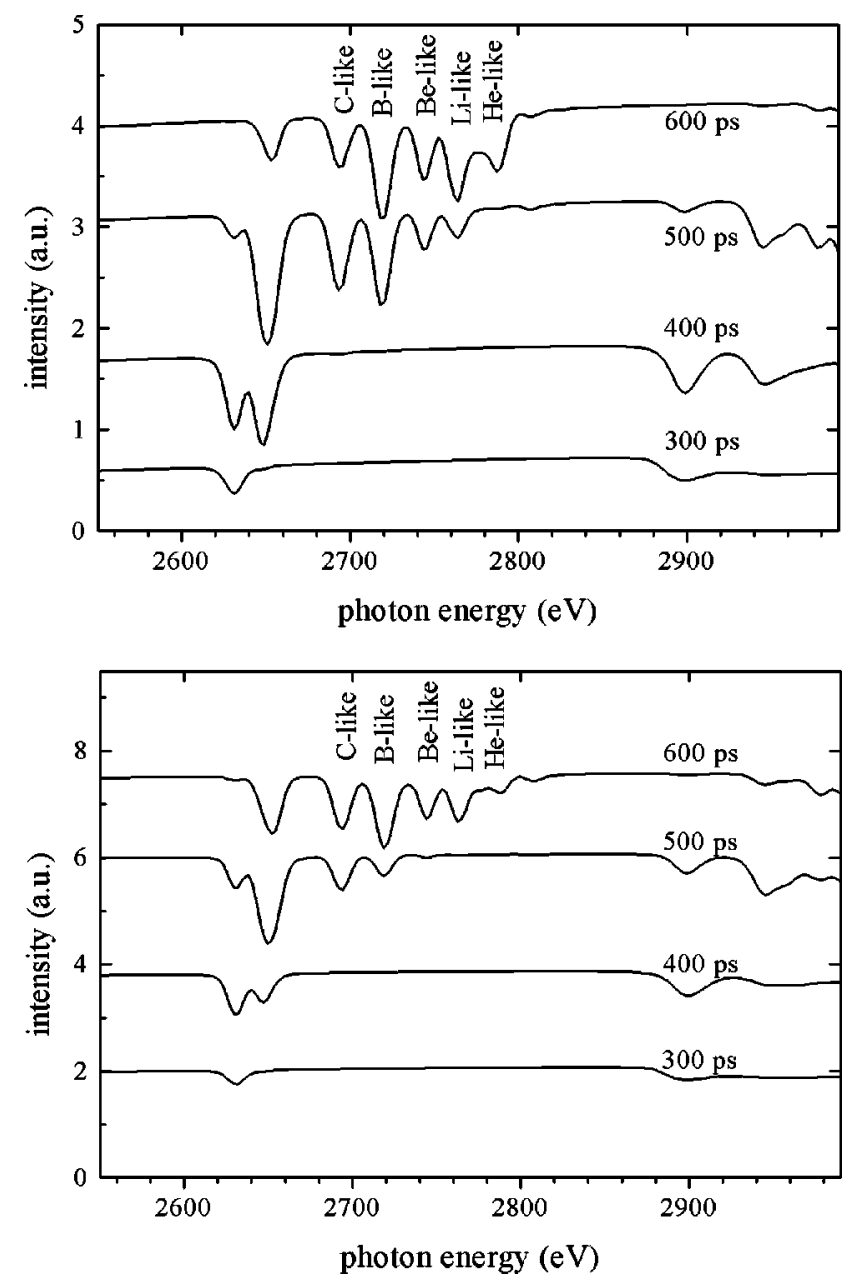

FIG. 3. Spectral simulations postprocessed from the hydrodynamics simulations discussed in Sec. III and shown in Fig. 7, assuming the tracers in both the undoped and doped samples are at the same depth $(6.3 \mu \mathrm{m})$. The simulated spectra for the undoped samples are shown in the top panel and those for the doped sample are shown in the lower panel. Note the earlier turn-on times of a given tracer ionization stage in the undoped sample.

temperature, and having very similar time histories. Note that in the next section we discuss the halfraum radiation field modeling and show that the calculated drive temperatures are within the DANTE error bars. We diverted six additional beams onto the bismuth backlighter foil, with staggered timing, to produce a relatively temporally smooth backlighter spectrum.

The ablator samples were manufactured at General Atomics using glow discharge polymerization (GDP) to lay down a layer of plastic (doped or undoped) several tens of microns thick. This wafer of plastic was then coated with sodium chloride $(\mathrm{NaCl})$ to a thickness of approximately 4000 $\AA$. On top of this, another, thinner layer of plastic was deposited in the same GDP chamber as the first layer. The sample was then cut into roughly ten pieces and each was characterized in terms of its density and composition. The undoped samples were typically $54.6 \% \mathrm{H}, 42.2 \% \mathrm{C}$, and $3.3 \% \mathrm{O}$ with a density of $1.11 \mathrm{~g} \mathrm{~cm}^{-3}$. The germaniumdoped samples had $55.4 \% \mathrm{H}, 38.6 \% \mathrm{C}, 4.2 \% \mathrm{O}$, and $1.8 \%$ Ge with a density of $1.28 \mathrm{~g} \mathrm{~cm}^{-3}$. These are the compositions used to calculate the opacities shown in Fig. 1. We
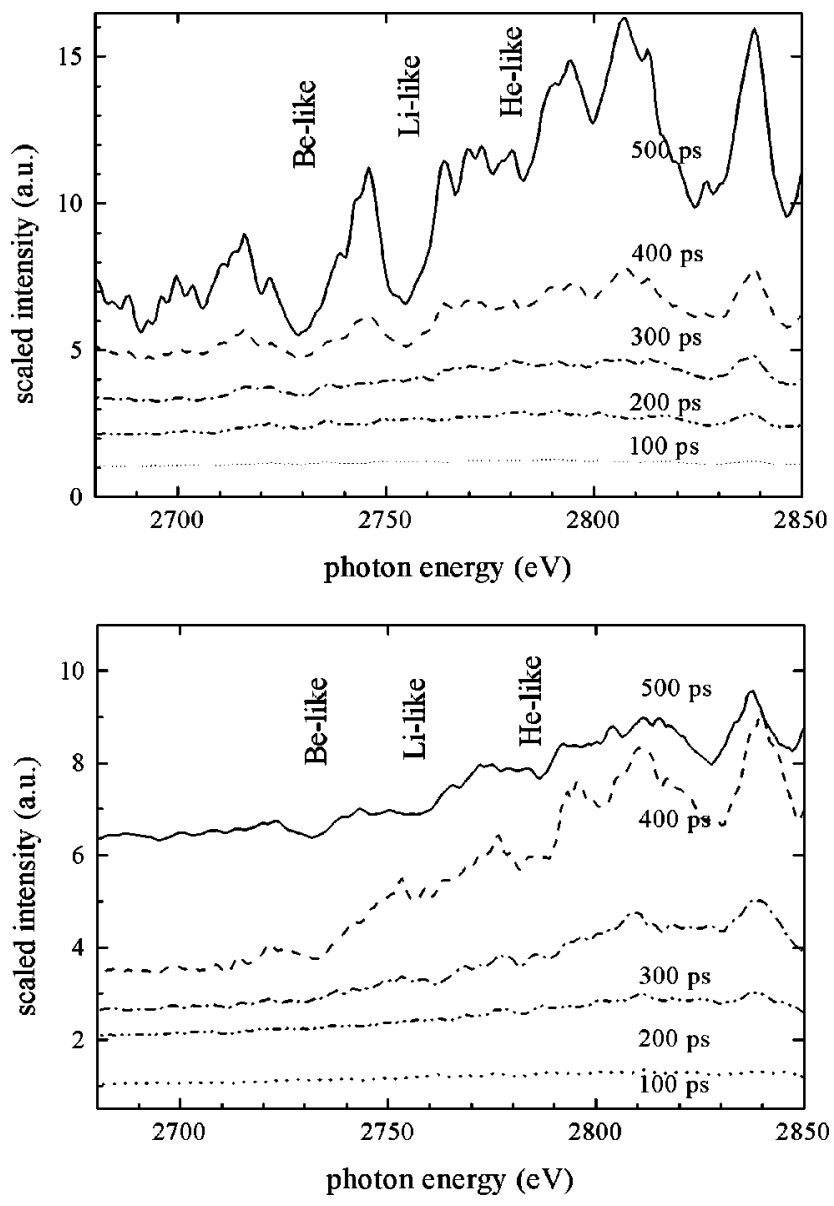

FIG. 4. Lineouts of the LLE x-ray spectrometer (LXS) spectra in the region of the $\mathrm{Cl} \mathrm{K}_{\alpha}$ features. Shot 19526 , with the undoped sample, is shown on the top and shot 19528 , with a doped sample is shown on the bottom. For both shots we show lineouts from the same times; every $100 \mathrm{ps}$ from 100 to $500 \mathrm{ps}$. These spectral lineouts have been scaled by constant additive factors for clarity of display. Note, finally, that the backlighter signal is relatively weaker at early times and also at lower photon energies, especially in the germanium-doped sample, making it difficult to see ionization stages lower than $\mathrm{Cl}^{+13}$, or Be-like.

discuss two different shots in this paper. In shot 19526 the tracer layer was $6.3 \mu \mathrm{m}$ from the surface of the undoped sample and in shot 19528 the tracer layer was $4.1 \mu \mathrm{m}$ from the surface of the doped sample.

In Fig. 4 we show time-dependent spectra measured on these two shots. Although there is a fair amount of (emission) structure in the backlighter spectrum, absorption signals from at least three ionization stages of chlorine can be seen turning on after the backlighter signal itself, and with the lower ionization stages appearing before the higher ones (this last effect is more apparent in the undoped sample, shot 19526). The turn-on time is slightly earlier in the undoped sample than in the doped sample, which is consistent with the qualitative expectations of the effect of the dopant on the Marshak wave velocity. Note the Be-like feature may appear weakly in the $300 \mathrm{ps}$ lineout in the undoped sample (top panel), and by 400 ps the Be-like and Li-like features are strongly present.

In the doped sample (lower panel of Fig. 4), the spectral signal becomes visible at $400 \mathrm{ps}$, and the ionization balance 
in this sample is weighted toward higher ion stages than in the undoped sample. The spectral signal is weaker overall in the doped sample because of the effect of the added opacity from the germanium dopant. It should be borne in mind that the tracer in the doped sample in the experiments is $2 \mu \mathrm{m}$ closer to the surface than the tracer in the undoped sample, so this modest time lag between the doped and undoped samples represents a significantly lower Marshak wave velocity in the germanium-doped plastic than in the undoped plastic. In other words, the tracer spectral signal turns on later in the doped sample, despite the fact that the tracer is located more than 30 percent closer to the surface in that sample.

\section{MODELING}

To understand the experimental results, and to infer the physical conditions in the halfraum and the ablator, we perform three stages of numerical modeling: (1) View-factor calculations, which provide the temperature structure (both spatial and temporal) within the halfraum as well as the incident radiation flux as a function of time on all surfaces, including the ablator sample; (2) hydrodynamics simulations of the interaction between the time-dependent radiation field in the halfraum and the ablator sample; and (3) spectral postprocessing of the hydrodynamics calculations. Associated atomic modeling is carried out to provide inputs at stages (2) and (3).

We used the VISRAD view-factor code ${ }^{13}$ to do the first step in the modeling. This code has been used to model both laser hohlraum and Z-pinch experiments, and accurately reproduced data from similar, previously published OMEGA hohlraum experiments. ${ }^{14,15}$ VISRAD is a time-independent code that assumes albedos and $\mathrm{x}$-ray conversion efficiencies for each surface in a simulation, which are assumed to emit as blackbodies. Temperature-dependent albedos are calculated for the gold hohlraums separately in a hydrodynamics code, using detailed opacities, the recorded beam power profiles are used, and with a value for the (time-dependent) $\mathrm{x}$-ray conversion efficiency (XCE) assumed, this modeling is fully constrained, with no adjustable parameters. This modeling procedure has been quite successful in reproducing the observed conditions in laser hohlraums. ${ }^{15}$

In Fig. 5 we show the good match obtained between VISRAD view-factor modeling of the wall reemission in earlier OMEGA hohlraum experiments and DANTE measurements. We also show in that same figure the midplane drive temperature from the same modeling and note that it is significantly different than the wall reemission temperature profile. This difference in the DANTE and drive temperatures makes sense because DANTE sees only hot spots and wall reemission, while a package at the midplane of a hohlraum sees hot spots and wall but also sees the cold LEHs as well. The same will be true for a package on the end of a halfraum. The percentage reduction in temperature at the package versus that seen by DANTE will depend on the relative distances of spots and LEH from the package, which differ between hohlraum and halfraum geometry. These results demonstrate the need for modeling of the drive conditions on

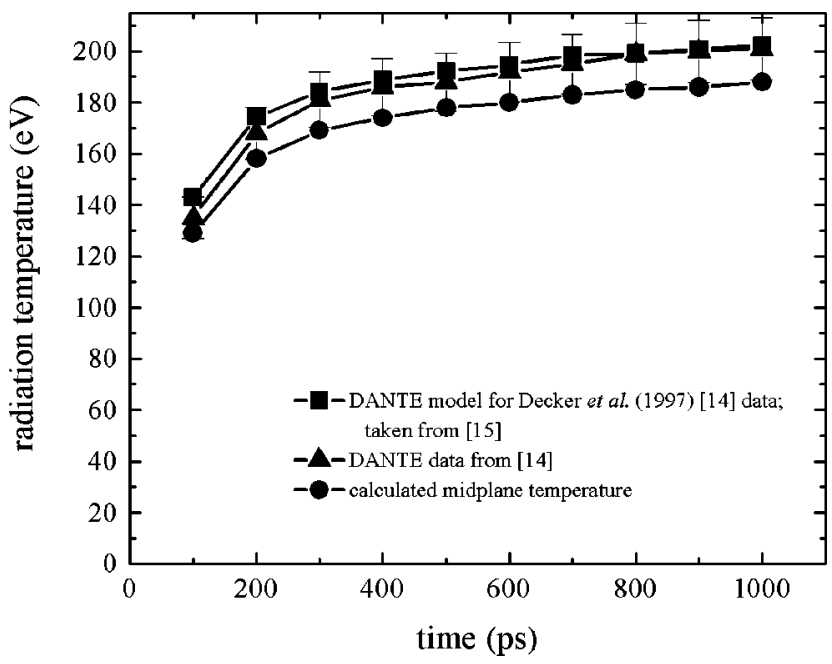

FIG. 5. Results of view-factor simulations of previously published hohlraum data from OMEGA. The filled squares are the simulated DANTE temperatures and the filled triangles are the measured DANTE temperatures for the OMEGA hohlraum shots discussed in Refs. 14 and 15. There is very good agreement between these data and the model, for which it should be noted, there were no adjustable parameters. The filled circles are the midplane temperatures from the same simulations. Note that these differ significantly from the DANTE wall reemission temperatures because of the different contributions from hot spots, wall reemission, and cold LEH for the two different views.

an experimental package, even when DANTE or other wall reemission measurements are made. And they also demonstrate, through the good agreement between the DANTE measurements and the view-factor modeling, the accuracy of the modeling technique.

In Fig. 6 we show the results of similar view-factor modeling for the OMEGA experiments we report on in this paper. This modeling was carried out in exactly the same way as that described above, using the same temperature-dependent gold albedo and time-dependent XCE ( 0.55 after 200 ps, ramping up from 0.2 initially) as was used to successfully

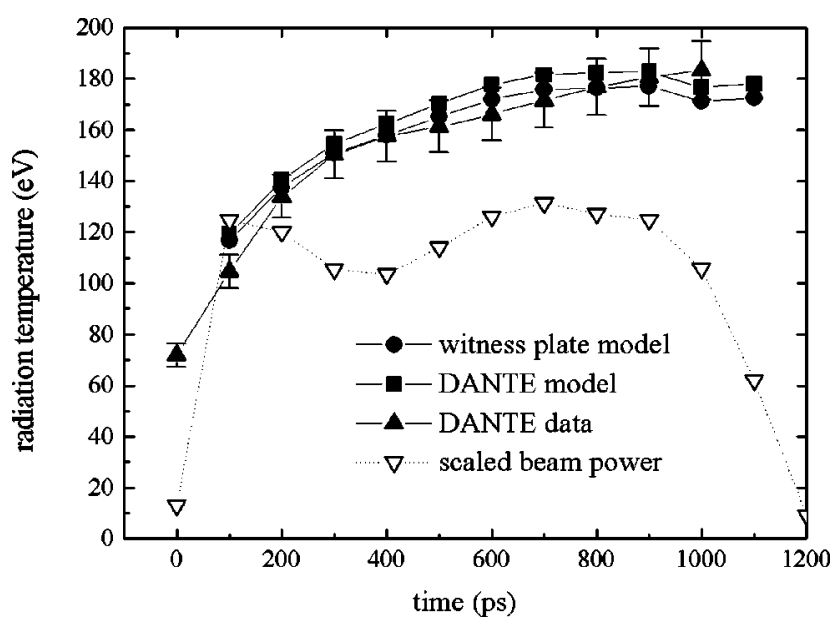

FIG. 6. Results of view-factor simulations of our OMEGA halfraum experiments. The filled squares are the simulated DANTE temperatures and the filled triangles are the measured DANTE temperatures. The filled circles are the calculated time-dependent drive temperature onto the sample, which is used as input to the subsequent hydrodynamics models. We also show the (scaled) laser beam powers from shot 19526. 

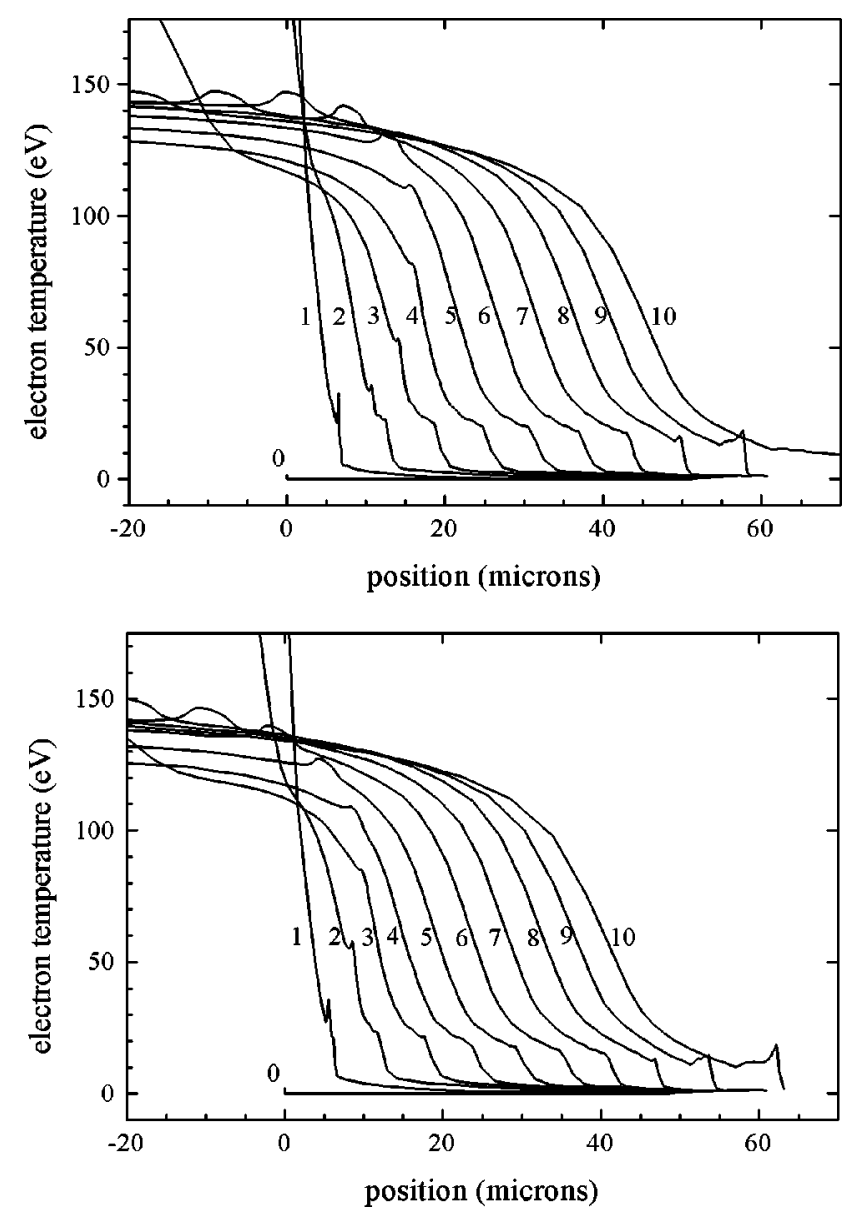

FIG. 7. Hydrodynamics simulations of the undoped (top) and doped (bottom) samples, with the temperature profiles shown at 11 different times, from $t=0$ to $t=1 \mathrm{~ns}$. Each time is labeled in units of $100 \mathrm{ps}$. Note that the Marshak wave velocity is slower in the doped sample. A shock wave, moving ahead of the radiation wave, is visible in the simulations. The location of the tracer layer can be discerned, as the tracer is slightly hotter than the surrounding plastic. Finally, note the hot ablated plasma on the left (radiation is from the left). At early times it is especially hot due to the deposition of scattered laser light onto the ablator sample.

match the data in the earlier hohlraum experiments. Our calculations also predict the wall reemission temperatures seen by DANTE, which are within the error bars of the absolutely calibrated DANTE measurements.

The time-dependent $\mathrm{x}$-ray spectrum incident on the ablator sample calculated from the view-factor modeling was next used as input to a hydrodynamics simulation performed with HELIOS. ${ }^{16}$ This one-dimensional, planar, Lagrangian simulation used multigroup opacities for both the plastic and the tracer calculated for the purpose. SESAME equations of state were used, and scattered laser light onto the ablator sample was also included. The scattered laser intensity was calculated in the VISRAD simulations to be roughly 100 TW $\mathrm{cm}^{-2}$ for the first $200 \mathrm{ps}$, falling off rapidly afterward. In Fig. 7 we show representative temperature profiles from these simulations. The shock wave and radiation wave can easily be seen, with the slower velocity in the doped sample apparent in these time series. We note here that we shifted the radiation drive calculated with VISRAD by 100 ps to account for uncertainties in the relative zero points of the
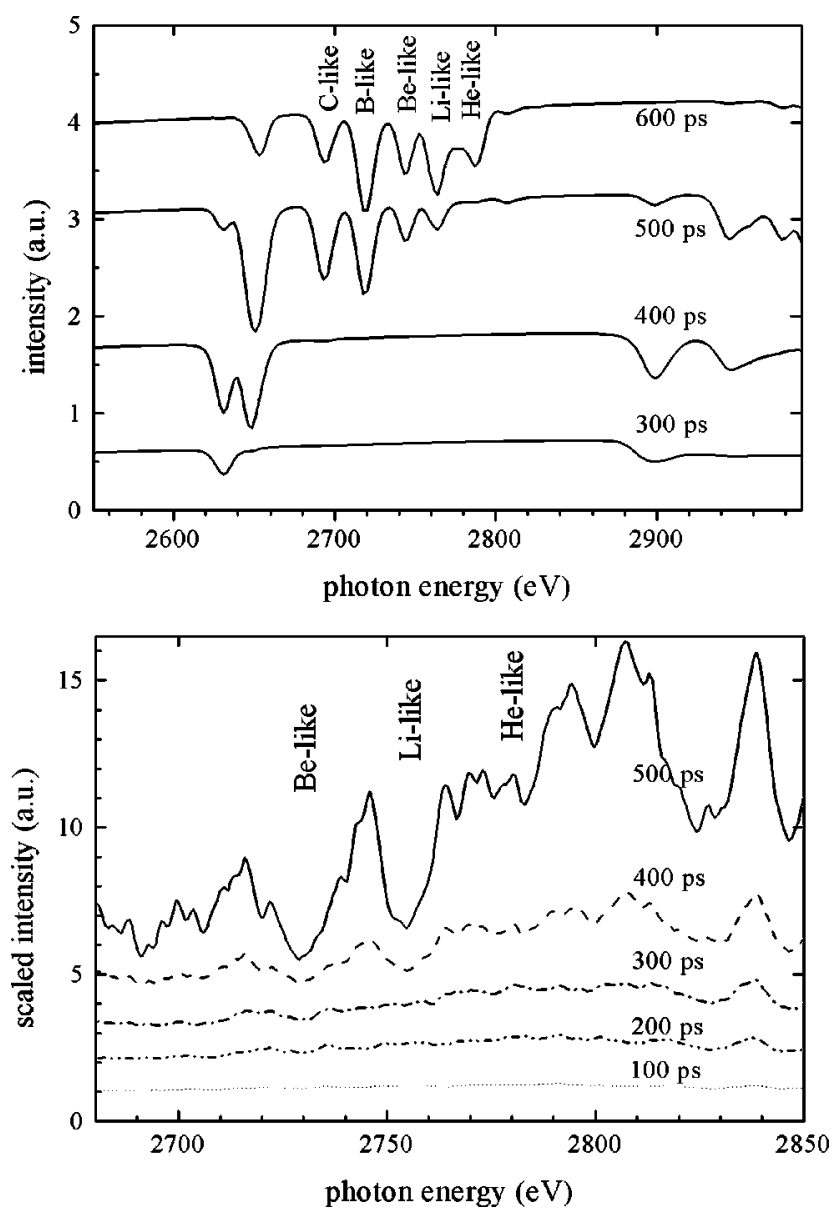

FIG. 8. Spectral simulations postprocessed from the hydrodynamics simulations shown in Fig. 7 (top) and the LXS data from shot 19526 (bottom), with the undoped target and a tracer depth of $6.3 \mu \mathrm{m}$. Note that the $x$-axes ranges are different in the model and data plots.

DANTE timing and the LXS timing. The apparent turn-on of the LXS-measured spectra should be somewhat later than the DANTE turn-on time, as the LXS is sensitive only to hard $\mathrm{x}$ rays (between 2 and $3 \mathrm{keV}$ ).

Ultimately, we synthesized the time-dependent chlorine absorption spectra via a series of time-independent calculations of the excitation/ionization and radiation transport through the hot (and cold) plasma derived from the HELIOS simulations. We custom compute atomic models for the ablator and tracer materials, which are used as inputs to the spectral calculations, which we carried out with the Spect3d code. ${ }^{17}$ We use over 1100 levels in our chlorine model, and assume local thermodynamic equilibrium (LTE) for the calculations we present here [using a time-independent collisional-radiative equilibrium (CRE) model gave very similar results]. We present spectral simulations, in Figs. 8 and 9, for the two samples shot. We stress that the earlier turn-on times seen in the doped sample in these simulations are entirely due to the smaller tracer depths. In these figures, we again show the data lineouts for comparison.

These spectral simulations show that the Be-like and higher ion stages become visible slightly earlier in the doped sample than in the undoped sample (400 ps versus $500 \mathrm{ps).}$ In both simulations a progression from lower to higher ion- 

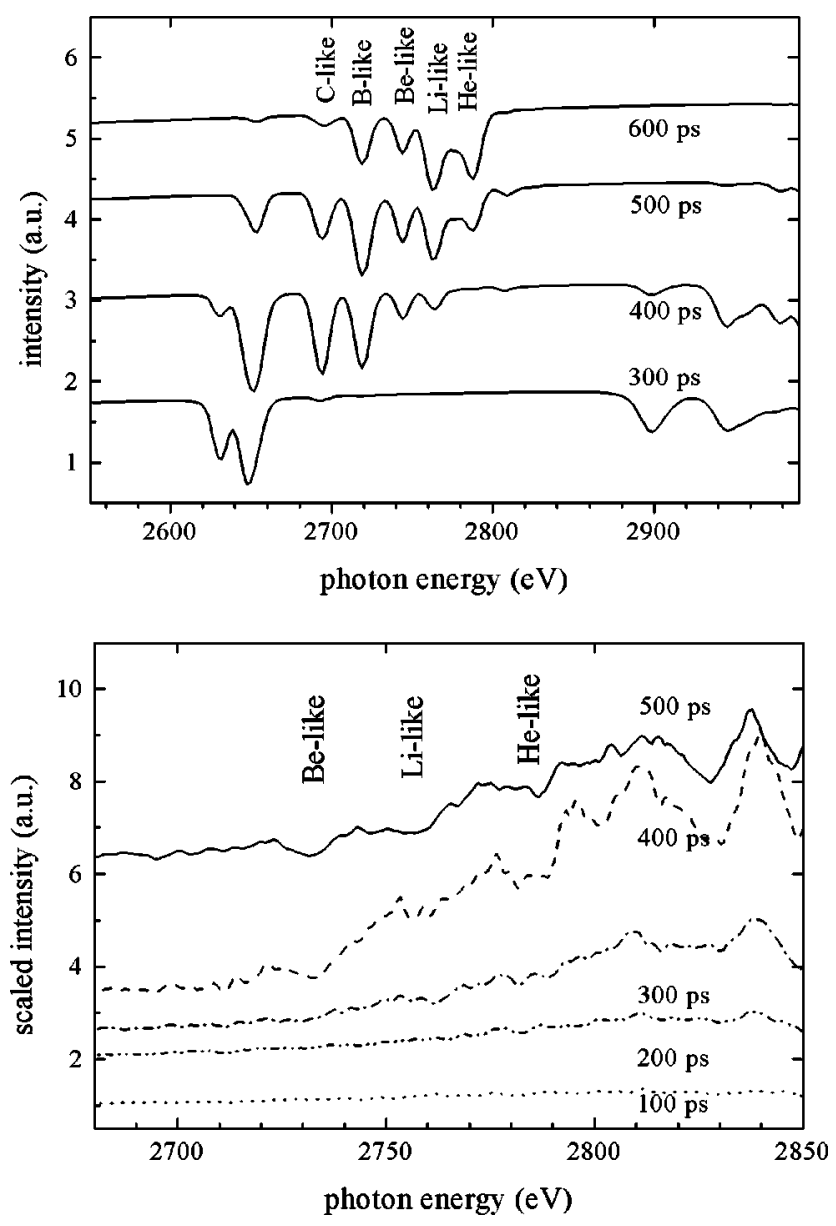

FIG. 9. Spectral simulations postprocessed from the hydrodynamics simulations shown in Fig. 7 (top) and the LXS data from shot 19528 (bottom), with the doped target and a tracer depth of $4.1 \mu \mathrm{m}$. Note that the $x$-axes ranges are different in the model and data plots.

ization stages can be seen, reflecting the increasing tracer temperature with time. These turn-on times in the simulations can be compared to those in the data: In the data from the undoped shot (Fig. 8) Be-like turns on at $400 \mathrm{ps}$ or slightly earlier versus $500 \mathrm{ps}$ in the model. In the data from the doped shot (Fig. 9) Be-like turns on at 400 ps, which is in good agreement with the model results.

\section{DISCUSSION}

Despite the noisiness of the spectral data (due to emission spectral structure in the Bi backlighter), we have detected the tracer absorption signals in the two samples. The detection is weaker in the doped sample, due to the lower signal-to-noise in the spectrum transmitted through the higher-opacity material. The tracer turn-on times observed in the undoped sample are slightly earlier than in the doped sample, especially if one looks at the He-like feature, which is relatively robust in the $400 \mathrm{ps}$ lineout for the doped sample, but only just beginning to appear in the 500 ps lineout in the undoped sample.

It seems likely that our nondetection of ion stages lower than Be-like can be understood in terms of the shape of the underlying backlighter pseudocontinuum, which is significantly weaker at energies below about $2750 \mathrm{eV}$, as has been seen in other experiments. ${ }^{10}$ The tracer turn-on time observed in the undoped sample is somewhat earlier than our modeling predicts, even under the most conservative assumptions. The appearance of Be-like absorption at $300 \mathrm{ps}$ is roughly $200 \mathrm{ps}$ earlier than in the simulations. Taken at face value, the effect of the germanium dopant in the experiments is thus even more dramatic than what is expected from our calculations.

It is conceivable that the chlorine tracer was closer to the surface of the ablator samples than we assumed, either due to uncertainty in the fabrication and characterization process or due to the possible migration of chlorine from the tracer salt into the plastic after fabrication. If so, this would seem to have affected the undoped sample more than the doped sample, based on the fact that it was the undoped sample for which there was a discrepancy in the calculated and measured tracer turn-on times. In the future, fabrication of tracer layers using chlorinated plastic rather than salt could be attempted. It is also possible the spatial nonuniformity in the tracer salts (due, for example, to crystallization) could have caused the tracer absorption signal to be weaker than it might otherwise have been. Using chlorinated plastic tracer layers, rather than salt, in the future might therefore also improve the signal strength.

In any case, if we infer the Marshak wave velocity from the experimental results we can tentatively conclude the following: If we take the turn-on time of the tracer signal to be $300 \mathrm{ps}$ in the undoped ablator and $400 \mathrm{ps}$ in the doped ablator (for the appearance of Be-like absorption), then the Marshak wave moves through an areal mass of 7 $\times 10^{-4} \mathrm{~g} \mathrm{~cm}^{-2}$ in $300 \mathrm{ps}$ for the undoped ablator and 5 $\times 10^{-4} \mathrm{~g} \mathrm{~cm}^{-2}$ in $400 \mathrm{ps}$ for the germanium-doped ablator. Expressed in units of areal mass per time, the effect of the ablator dopant is thus to reduce the Marshak wave velocity by almost $50 \% \quad\left(2.3 \times 10^{-3} \mathrm{~g} \mathrm{~cm}^{-2} \mathrm{~ns}^{-1}\right.$ versus 1.3 $\left.\times 10^{-3} \mathrm{~g} \mathrm{~cm}^{-2} \mathrm{~ns}^{-1}\right)$.

Finally, it is clear that the detailed experimental modeling is crucial for interpreting the relatively complex data from these experiments. And, that in conjunction with such modeling, point-projection absorption spectroscopy of buried tracer layers is a promising - but challenging-diagnostic technique for inferring the localized properties of plasmas in ICF environments.

\section{ACKNOWLEDGMENTS}

We wish to thank Rick Olson, John Oertel, Jim Bailey, Tom Boehly, Wang Ping, Pamela Woodruff, Igor Golovkin, Russ Wallace, Bob Turner, Abbas Nikroo, Pete Gobby, Dave Hoffman, Jerry Kulcinski, John Soures, the OMEGA operations and target fabrication teams, especially Sam Morse and Greg Pien, for their help with target fabrication, diagnostics, modeling, and discussion of various science issues.

We also are grateful to the NLUF program for support through Grant DE-FG03-OOSF22023, and General Atomics, Livermore, and LLE for target fabrication and assembly. 
${ }^{1}$ O. L. Landen, C. J. Keane, B. A. Hammel, W. K. Levedahl, P. A. Amendt, J. D. Colvin, M. D. Cable, R. Cook, T. R. Dittrich, S. W. Haan et al., Phys. Plasmas 3, 2094 (1996).

${ }^{2}$ T. R. Dittrich, S. W. Haan, M. M. Marinak, S. M. Pollaine, D. E. Hinkel, D. H. Munro, C. P. Verdon, G. L. Strobel, R. McEachern, R. C. Cook et al., Phys. Plasmas 6, 2164 (1999).

${ }^{3}$ R. E. Olson, J. L. Porter, G. A. Chandler, D. L. Fehl, D. O. Jobe, R. J. Leeper, M. K. Matzen, J. S. McGurn, D. D. Noack, L. E. Ruggles et al., Phys. Plasmas 4, 1818 (1997).

${ }^{4}$ J. P. Jadaud, E. de Sainte-Claire, M. A. Blain, D. Desenne, A. Richard, M. R. L. Glohaec, O. Landen, H. Kornblum, T. Orzechowski, R. Wallace et al., Bull. Am. Phys. Soc. 41, 1389 (1996).

${ }^{5}$ T. S. Perry, R. A. Ward, D. R. Bach, R. J. Doyas, B. A. Hammel, D. W. Phillion, H. N. Kornblum, J. M. Foster, P. A. Rosen, R. J. Wallace et al., J. Quant. Spectrosc. Radiat. Transf. 51, 273 (1994).

${ }^{6}$ T. S. Perry, S. J. Davidson, F. J. D. Serduke, D. R. Bach, C. C. Smith, J. M. Foster, R. J. Doyas, R. A. Ward, C. A. Iglesias, F. J. Rogers et al., Phys. Rev. Lett. 67, 3784 (1991).

${ }^{7}$ T. S. Perry, P. T. Springer, D. F. Fields, D. R. Bach, F. J. D. Serduke, C. A. Iglesias, F. J. Rogers, J. K. Nash, M. H. Chen, B. G. Wilson et al., Phys. Rev. E 54, 5617 (1996).

${ }^{8}$ C. A. Back, S. H. Glenzer, K. Estabrook, R. L. Kauffman, O. L. Landen, B. J. MacGowan, L. V. Powers, T. D. Shepard, and G. F. Stone, Bull. Am. Phys. Soc. 41, 1600 (1996).

${ }^{9}$ C. A. Back, O. L. Landen, R. E. Turner, J. A. Koch, B. A. Hammel, J. J. MacFarlane, D. H. Cohen, and T. J. Nash, Bull. Am. Phys. Soc. 42, 1992 (1997).

${ }^{10}$ C. Chenais-Popovics, C. Fievet, J. P. Geindre, J. C. Gauthier, E. JucKoenig, J. F. Wyart, H. Pepin, and M. Chaker, Phys. Rev. A 40, 3194 (1989).
${ }^{11}$ T. R. Boehly, D. L. Brown, R. S. Craxton, R. L. Keck, J. P. Knauer, J. H. Kelly, T. J. Kessler, S. A. Kumpan, S. J. Loucks, S. A. Letzring et al., Opt. Commun. 133, 495 (1997).

${ }^{12}$ H. N. Kornblum, R. L. Kauffman, and J. A. Smith, Rev. Sci. Instrum. 57, 2179 (1986)

${ }^{13}$ J. J. MacFarlane, J. Quant. Spectrosc. Radiat. Transf. 81, 287 (2003).

${ }^{14}$ C. Decker, R. E. Turner, O. L. Landen, L. J. Suter, P. Amendt, H. N. Kornblum, B. A. Hammel, T. J. Murphy, J. Wallace, N. D. Delamater et al., Phys. Rev. Lett. 79, 1491 (1997).

${ }^{15}$ J. J. MacFarlane, astro.swarthmore.edu/prism/visrad-benchmark.pdf; see EPAPS Document No. E-PHPAEN-11-911405 for "Visrad benchmark calculation: OMEGA Hollraum radiation temperatures." A direct link to this document may be found in the online article's HTML reference section. The document may also be reached via the EPAPS homepage (http:// www.aip.org/pubservs/epaps.html) or from ftp.aip.org in the directory /epaps/. See the EPAPS homepage for more information.

${ }^{16}$ I. E. Golovkin, J. J. MacFarlane, P. R. Woodruff, L. A. Welser, D. L. McCrorey, R. C. Mancini, and J. A. Koch, in the Proceedings of the Third International Conference on Inertial Fusion Sciences and Applications 3 (to be published by Elsevier).

${ }^{17}$ J. J. MacFarlane, I. E. Golovkin, P. R. Woodruff, D. R. Welch, B. V. Oliver, T. A. Mehlhorn, and R. B. Campbell, in the Proceedings of the Third International Conference on Inertial Fusion Sciences and Applications 3 (to be published by Elsevier).

${ }^{18}$ See EPAPS Document No. E-PHPAEN-11-911405 for a color version of the lower panel of Fig. 2. A direct link to this document may be found in the online article's HTML reference section. The document may also be reached via the EPAPS homepage (http://www.aip.org/pubservs/ epaps.html) or from ftp.aip.org in the directory /epaps/. See the EPAPS homepage for more information. 